\title{
Towards a knowledge management framework for crossing knowledge boundaries in agricultural value chain
}

Mileva Boshkoska, B

http://hdl.handle.net/10026.1/11322

10.1080/12460125.2018.1468173

Journal of Decision Systems

Taylor \& Francis

All content in PEARL is protected by copyright law. Author manuscripts are made available in accordance with publisher policies. Please cite only the published version using the details provided on the item record or document. In the absence of an open licence (e.g. Creative Commons), permissions for further reuse of content should be sought from the publisher or author. 


\title{
Towards a knowledge management framework for crossing knowledge boundaries in agricultural value chain
}

\author{
Biljana Mileva Boshkoska, \\ “Jožef Stefan” Institute, Ljubljana, Slovenia and \\ Faculty of Information Studies, Novo mesto, Slovenia \\ Shaofeng Liu, Huilan Chen
}

Plymouth Business School, University of Plymouth, Plymouth, UK

\section{Corresponding author:}

Biljana Mileva Boshkoska, “Jožef Stefan” Institute, Jamova cesta 39, SI-1000 Ljubljana, Slovenia

e-mail: biljana.mileva@ijs.si

\section{Abstract}

The purpose of the paper is to propose a framework for the development of a decision support system in order to evaluate the knowledge boundaries in agricultural value chain. Knowledge boundaries exist due to differences in the way we work, share our knowledge, expertise, different organisational culture, etc. In this paper we identify the most common knowledge boundaries that are reported in the literature, and propose a general framework for a preparation of a decision support system to evaluate the existing knowledge boundaries. In particular, we are interested in identifying the knowledge boundaries in agricultural value chain, evaluating them and providing possible solutions of crossing them. It is a two-step method: firstly, a semi-automatic ontology is generated using the freely available tool OntoGen, which we use to define the most commonly reported concepts in crossing knowledge boundaries, and then, based on the obtained ontology, we propose a decision support system for evaluation of the level to which the boundaries exist.

Keywords: Knowledge management, knowledge boundaries, decision support system, agricultural value chain, DEX 


\section{Introduction}

Knowledge management has been established as a subject in its own right for decades. Integrating knowledge management into decision support systems has been investigated by many scholars which resulted in the emergence of expert systems and knowledge-based decision support systems (Zarate \& Liu, 2016). One of the key challenges of knowledge management and implementing knowledge management for decision support represent the barriers created by knowledge boundaries whether existing between different domains, different practitioners' groups, or people with different level of knowledge even within the same domain and group, such as between novices and experienced practitioners (Carlile, 2002). This paper reports part of the research work associated with the EU Horizon 2020 project RUC-APS (Enhancing and implementing Knowledge based ICT solutions within high Risk and Uncertain Conditions for Agriculture Production Systems, https://ruc-aps.eu/), aiming at the development of a framework for preparation of a decision support system for crossing the knowledge boundaries in the domain of agricultural value chain. The question that we try to answer is how can knowledge flow and mobilize among the whole agricultural value chain, from farm to fork, by freely crossing different stages of the value chain (on the vertical dimension), and crossing different bodies even at the same stage of the chain but with different level of knowledge (on the horizontal dimension). The paper is focused on the proposal of a framework for the development of a decision support system that can help evaluate knowledge management boundaries in agricultural value chain. The knowledge required in agricultural domain is varied. Typically this knowledge is situated within the boundaries of a specific level of the value chain, for example, farmers, cooperatives, food processors, wholesalers, retailers and consumers (Chen, Liu, \& Oderanti, 2017) as represented in Figure 1. 


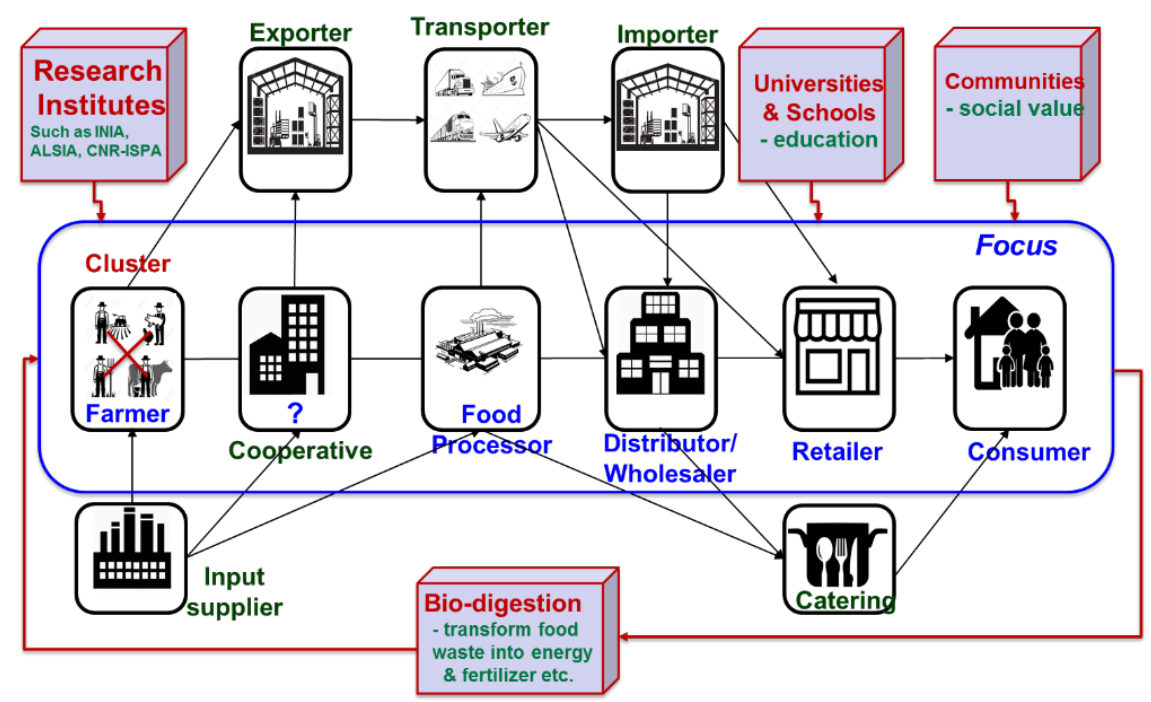

Figure 1 Knowledge sharing in value chain

It is therefore important that the knowledge assets, which are situated at one level, are linked to another. Boundaries are defined in many domains, hence literature overview comes from many sources. Exploring the boundaries that occur in knowledge, regarded as knowledge boundaries, (Tell, Berggren, Brusoni, \& Van de Ven, 2017) identify five major categories: individual, domain-specific, task-oriented, spatial, and temporal. Knowledge boundaries (Carlile, 2002), (Carlile, 2004) have been studied in different areas (Swart \& Harvey, 2011), however only a few studies in the domain of agriculture have been conducted so far (Hartwich, Pérez, Ramos, \& Soto, 2007).

\section{Methodology}

We propose a two-step framework for development of a decision support system (DSS) for evaluation of the level of knowledge boundaries in agriculture. In the first step, we conduct a literature overview to define the most frequently used terms (keywords) based on which we develop an ontology of knowledge boundaries. In the second step, we use the obtained ontology to define the attributes of the decision support system for evaluation of the knowledge boundaries. Both steps are defined in continuation. 


\section{Defining a knowledge boundary ontology based on literature review}

From the Web of Science database we extracted papers that use the keyword "knowledge boundaries” which lead to 63 articles, published in the period from 2002 - 2018, most of which were published in the area of management, business and information sciences as shown in Figure 2. None of the published papers were categorized in the agricultural domain. When working with many documents obtained from standard search engines, it is hard to comprehend and process all the information contained in them. This is a result of the search process which is mainly based on word matching and does not take into account the structure of the document. To overcome this problem, we employed the OntoGen software tool for construction of an ontology for knowledge boundaries based on automatic topic extraction from abstracts of the downloaded papers (Fortuna, Grobelnik, \& Mladenić, 2005). The process of selecting the abstracts was followed by formatting them in the required format for the OntoGen software, which was afterwards used to develop the ontology depicted in Figure 3.

Figure 2 Distribution of published papers for knowledge boundaries over different categories. Other are categories in which 2 or less papers were published in Web of Science. 


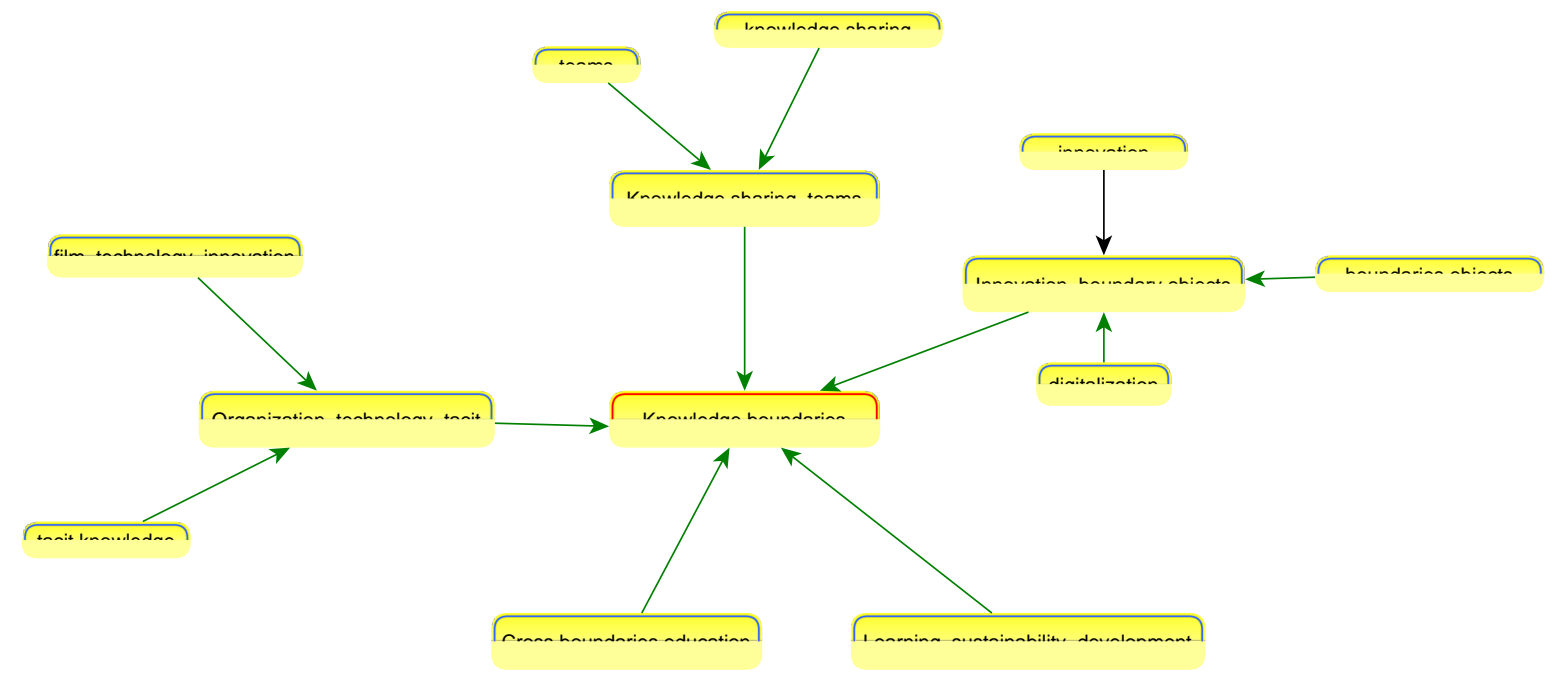

Figure 3 Concepts that are most commonly used with knowledge boundaries

From the ontology, the following most frequently researched concepts were extracted as sub topics of the knowledge boundaries:

1. Learning, sustainability, development (networks).

2. Cross boundaries education (networks).

3. Innovation, boundary objects (knowledge types).

4. Knowledge sharing, teams (networks).

5. Organization, technology, human/tacit knowledge (knowledge types, networks).

In the first concept, Learning, sustainability, development, research topics from eight articles cover different social networks which are formed with the purpose of forming an environment for learning (schools, projects, workplace, etc.) as well as the formation of cross-border networks and interactions which aim to guarantee the sustainable development of the obtained knowledge in the projects.

The second concept, Cross boundaries education, described in five of the examined papers, generalizes the crossing of boundaries so that the curriculum knowledge (academically gained) can be used both for work and academic requirements (Garraway, 2010), (Young \& Muller, 2010). In particular, four learning mechanisms are defined for crossing boundaries: 
identification, coordination, reflection, and transformation (Akkerman \& Bakker, 2011), (Hong \& Snell, 2015), (Barley, 2015).

The next concept, Knowledge sharing, teams, can be further divided into two sub concepts: Knowledge sharing, and teams. The sub category Knowledge sharing groups seven documents which deal with knowledge boundaries at newly emerging interfaces for knowledge sharing, knowledge sharing through learning, in particular explorative and exploitative knowledge sharing (Im \& Rai, 2008), and behaviour of groups that deal with the contradiction among distributed knowledge in boundary-spanning collaborative processes (Gasson, 2005).

In the sub category Teams, the examined nine papers research how to cross the boundaries between different team members, or in particular team leaders. The main boundaries are associated with different knowledge backgrounds of the team members' coming from various disciplines (Fitzgerald \& Rowley, 2015), (Lee, Min, \& Lee, 2017), (Wannenmacher \& Antoine, 2016), when teams are faced with novelty, and co-location of research and development teams in multi-space environment (Majchrzak, More, \& Faraj, 2012), (Coradi, Heinzen, \& Boutellier, 2015).

The concept Innovation, boundary object, objects is divided into three sub categories. The first one, Innovation, focuses on the knowledge exchanges across knowledge boundaries in activities of different organisations, which aim to provide an innovation (Rehm \& Goel, 2015), (Smith, 2016), functioning of innovation clusters and usage of knowledge brokering activities to cross knowledge boundaries (Castro, 2015); and open innovations (Wilhelm \& Dolfsma, 2018), which deals with obtaining knowledge from distant knowledge sources. The second sub category is boundary objects, and it examines the following questions: what is the relationship between school and every day or common sense knowledge (Marheineke, Habicht, \& Moslein, 2016), and how to overcome three progressively complex knowledge 
boundaries in organizations/networks: syntactic, semantic, and pragmatic (Abraham, Aier, \& Winter, 2015), (Rau, Neyer, \& Moslein, 2012).

The category digitalization provides a research on the role of digital science in complex innovation and its ability to create new knowledge boundaries that concern central activities of innovation (Dougherty \& Dunne, 2012), (Alin, Iorio, \& Taylor, 2013).

The last concept, Organization, technology, human, comprises 14 documents divided into two sub categories: film, technology, innovation; and tacit knowledge. The first one provides insights of how organizations bridge the boundaries between the required technological knowledge found externally, and how they align the external knowledge gathering with the activities to their strategies associated with developing current and future capabilities. The second one, examines the industry - university links to provide a conceptual bridge between internal labour markets and network organizations, as well as identification of knowledge boundaries in projects and networks (Swart \& Harvey, 2011).

\section{Defining a decision support system for evaluation of knowledge boundaries}

Using the obtained ontology (Figure 3), we developed a general DSS for evaluation of the level of knowledge boundaries. We demonstrate that the same DSS can be applicable to the field of agriculture as well. The DSS was developed using the DEX method (Bohanec, Rajkovič, Bratko, Zupan, \& Žnidaršič, 2013), (Bohanec M. , 2015). DEX is a qualitative multi-attribute decision modelling methodology that uses rule-based expert systems to build decision models. When using DEX, the decision maker is requested to define attributes and relation among attributes in the form of "if-then" rules, leading to utility functions that represent experts’ opinions, preferences and/or knowledge. In DEX, several attributes are aggregated into one, and the aggregated attribute is propagated to the next higher hierarchical level of the model. That way, DEX model represents a decomposition of the decision problem 
into smaller, less complex sub problems that are easily understandable for the human mind. The DEX model consists of: attributes, scales of attributes (usually qualitative set of words ordered in a preferential way, such as: 'developed', 'partially developed, 'underdeveloped', etc.), hierarchy of attributes (that represent a decision tree), and decision rules (interpreted as “if-then” rules). The DEX method is implemented in DEXi software tool which we used for the development of the DSS. The attributes, scales of attributes, and hierarchy of attributes for evaluation of the level of knowledge boundaries for the proposed DSS are given in Figure 4. It is a hierarchical model, where the root "Knowledge boundaries" is evaluated based on the values of its descendant attributes: “Cross boundary education”, “Learning, sustainability, development, “Innovation, boundary objects”, “Knowledge sharing, teams”, and “Organization, technology, tacit”. For each aggregated attribute, a utility table is defined by which define the rules of aggregation from lower level attributes to higher level attributes. An example of a utility table is provided in Figure 5 for the attribute "Knowledge sharing, teams". The qualitative values of the attribute "Knowledge sharing, teams" are obtained by aggregating the values of the attributes “knowledge sharing” and “teams”. The aggregation values are given in the utility table in Figure 5 in which each row in the utility table can be represented as an easily understandable "if-then" rule. For the given example in Figure 5 we may derive the following rules:

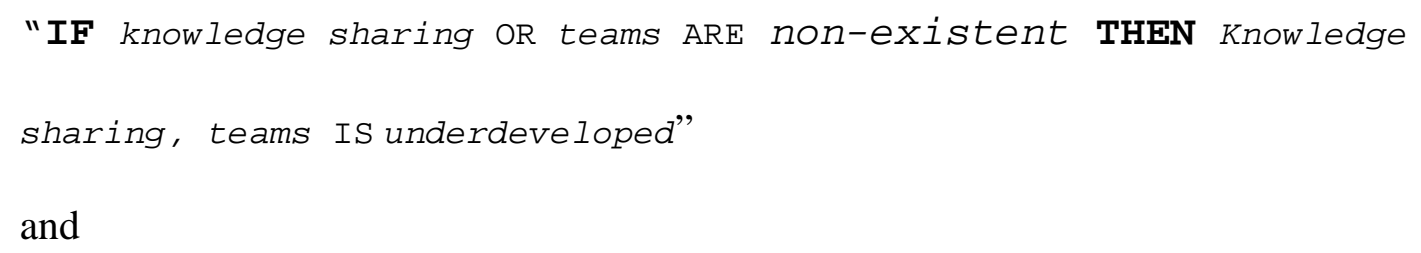




\section{Attribute}

\begin{tabular}{|c|}
\hline $\begin{array}{l}\text {-Learning, sustainability, develop } \\
\text {-Innovation, boundary objects } \\
\text {-innovation } \\
\text {-boundaries objects } \\
\text { _digitalization }\end{array}$ \\
\hline
\end{tabular}

Scale

strong; medium; weak; none

nonexistent; existent

underdeveloped; developed

underdeveloped; developed

underdeveloped; developed

undefined; defined

underappreciated; appreciated

underdeveloped; developed

nonexistent; existent

nonexistent; existent

underdeveloped; developed

underdeveloped; developed

underappreciated; appreciated

Figure 4 Attributes, scales of attributes, and hierarchy of attributes for evaluation of the level of knowledge boundaries

\section{knowledge sharing teams Knowledge sharing, teams}

\begin{tabular}{|c|c|c|c|}
\hline & $50 \%$ & $50 \%$ & \\
\hline $\begin{array}{l}1 \\
2\end{array}$ & ${ }_{*}$ nonexistent & " nonexistent & $\begin{array}{l}\text { underdeveloped } \\
\text { underdeveloped }\end{array}$ \\
\hline 3 & existent & existent & developed \\
\hline
\end{tabular}

A use case example for demonstration purposes of the obtained DSS is presented in Figure 6, where an evaluation of the knowledge boundaries are given for the use cases of Chile and south east China, chosen in the frames of the RUC-APS project. Attributes are colour coded so that the green colour represents the most preferred attribute value and the red colour represents the least preferred attribute value. Both use cases were developed with two experts from RUC-APS project, one of them originating from south east China region and the other working on the case of lean agro-food production in Chile. The final evaluation for knowledge boundaries in Chile and Southeast China are evaluated as medium and weak, respectively, as given in the row Knowledge boundaries in Figure 6. According to the evaluation model, the knowledge boundaries in Chile may be improved, if at least two of the attributes innovation, boundaries objects and/or firm, technology, tacit improve. The evaluation of the knowledge boundaries in Southeast China may be improved by improving the boundaries objects.

\begin{tabular}{lll} 
Attribute & Chile & Southeast China \\
\hline Knowledge boundaries & medium & weak \\
-Cross boundary education & existent & existent \\
- Learning, sustainability, development & developed & developed \\
-Innovation, boundary objects & underdeveloped & underdeveloped
\end{tabular}




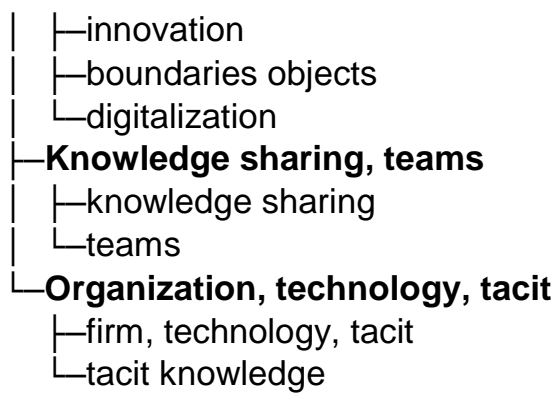

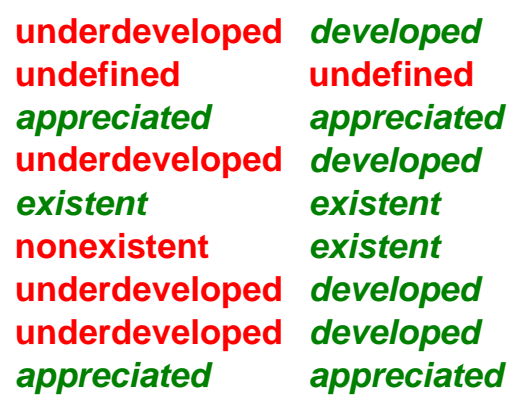

Figure 6 Use case examples for evaluation of knowledge boundaries in Chile and Southeast China

\section{The evaluation of the two-step framework and the proposed decision support system}

The proposed framework and the obtained decision support system have been evaluated by five knowledge management experts at the University of Plymouth, UK, who currently work in the agricultural sector and are involved in the RUC-APS project. All experts have background in agriculture and are PhD students in their final year of study. Their main research in RUC-APS project is focused on exploration of horizontal and vertical knowledge exchange mechanisms across the agricultural value chain. For evaluation purposes, in a semi structured interview, we thoroughly presented the approach we have taken for the development of the framework for crossing knowledge boundaries in agricultural value chain and the proposed DSS for evaluation of knowledge boundaries. Next we asked experts the following questions:

Is the framework understandable, and relevant for defining the crossing knowledge boundaries? Is the framework applicable for the knowledge boundaries in the agricultural sector? Do they think that the attributes obtained with OntoGen are relevant? Is the obtained hierarchy of the attributes in the proposed DSS correct? Would they change the classification scales of attributes in the proposed DSS? Do they find the obtained DSS applicable for evaluation of different case-studies in the agricultural sector useful?

During the evaluation interviews, all experts showed interest in the proposed framework and in the obtained ontology with OntoGen. They confirmed that the provided ontology reflects the used concepts in the area of knowledge management. Experts found the usage of OntoGen 
applicable to more specific areas of their work, such as lean supply chains in agriculture. Additionally, experts recognised the simplicity of the DEX model, its comprehensibility, and the straightforward explanation of the obtained evaluations.

All experts provided a positive opinion that the proposed framework for development of a DSS and agreed that it can be further used for agriculture systems in frames of the EU Horizon 2020 project RUC-APS. Two of the experts suggested to use the proposed DSS for evaluation of the knowledge boundaries in the use-cases that they have collected in the RUCAPS project. One expert suggested a possible improvement of the attribute "Knowledge sharing, teams” or even development of a separate sub model, that will consider other aspects of knowledge sharing, such as usage of digital technologies for knowledge sharing, expertise of the teams, and willingness of teams to cooperate.

\section{Conclusions}

In the framework of the RUC-APS project, we proposed framework for development of a decision support system for evaluation of the knowledge boundaries in agricultural value chain. It is a two-step approach based on OntoGen tool, used for generation of an ontology for knowledge boundaries, and the DEXi tool, used for expert modelling and preparation of the DSS for evaluation of the knowledge management boundaries. The framework was presented to five knowledge management experts who were able to formulate the problem of knowledge boundaries as a hierarchical structure of qualitative attributes, and were able to define all the necessary decision rules in the proposed model. All experts found the framework comprehensible, easy to use and applicable for their future work. In particular, experts showed great interest in using OntoGen, and provided the following suggestions for future work. Firstly, we have to revise the ontology concepts obtained with OntoGen by using full paper texts and include more database sources such as Science Direct and/or Springer Link. Secondly, based on the new developed concepts with OntoGen we may need to update the 
decision support system, including the suggested improvement and possible development of a sub-model regarding the attribute "Knowledge management, teams", which afterwards has to be validated in wider agricultural value chain scope without being restricted to the RUC-APS project. Finally, we will need to investigate more use cases from the RUC-APS project to confirm the usability of the proposed DSS for evaluation of the knowledge boundaries in the agricultural value chain.

\section{Acknowledgement}

The work reported in this paper has benefited from the RUC-APS project funded by European Commission under the Horizon 2020 Programme (H2020-MSCA-RISE Award No. 691249) as well as the operation "Support system for internationalization and transfer of knowledge” selected in the call for proposal Mobility of Slovenian higher education teachers 2017-2018, which is co-financed by the Republic of Slovenia and the European Union from the European Social Fund / European Regional Development Fund / Cohesion Fund.

\section{References}

Abraham, R., Aier, S., \& Winter, R. (2015). Crossing the Line: Overcoming Knowledge Boundaries in Enterprise Transformation. BUSINESS \& INFORMATION SYSTEMS ENGINEERING, 11.

Akkerman, S. F., \& Bakker, A. (2011). Boundary Crossing and Boundary Objects. Review of Educational Research, 132-169.

Alin, P., lorio, J., \& Taylor, J. (2013). Digital Boundary Objects as Negotiation Facilitators: Spanning Boundaries in Virtual Engineering Project Networks. PROJECT MANAGEMENT JOURNAL, 16.

Barley, W. (2015). Anticipatory Work: How the Need to Represent Knowledge Across Boundaries Shapes Work Practices Within Them. ORGANIZATION SCIENCE, 17.

Bohanec, M. (2015). DEXi: Program for multi-attribute decision making, User's manual, version 5.00. Ljubljana: Jožef Stefan Institute. IJS Report DP-11897. Ljubljana. Retrieved from http://kt.ijs.si/MarkoBohanec/pub/DEXiManual500.pdf

Bohanec, M., Rajkovič, V., Bratko, I., Zupan, B., \& Žnidaršič, M. (2013). DEX methodology: Three decades of qualitative multi-attribute modelling. Informatica, 49 - 54.

Carlile, P. (2002). A Pragmatic View of Knowledge and Boundaries: Boundary Objects in New Product Development. Organization Science, 442-455. 
Carlile, P. (2004). Transferring, Translating, and Transforming: An Integrative Framework for Managing Knowledge Across Boundaries. Organization Science, 555-568.

Castro, L. (2015). Strategizing across boundaries: revisiting knowledge brokering activities in French innovation clusters. JOURNAL OF KNOWLEDGE MANAGEMENT, 21.

Chen, H., Liu, S., \& Oderanti, F. (2017). A knowledge network and mobilisation framework for lean supply chain decisions in agri-food industry. International Journal of Decision Support System Technology, 37-48.

Coradi, A., Heinzen, M., \& Boutellier, R. (2015). Designing workspaces for cross-functional knowledge-sharing in R\&D: the "co-location pilot" of Novartis. JOURNAL OF KNOWLEDGE MANAGEMENT, 21.

Dougherty, D., \& Dunne, D. (2012). Digital Science and Knowledge Boundaries in Complex Innovation. ORGANIZATION SCIENCE, 18.

Fitzgerald, R., \& Rowley, C. (2015). How have Japanese multinational companies changed? Competitiveness, management and subsidiaries. ASIA PACIFIC BUSINESS REVIEW, 8.

Fortuna, B., Grobelnik, M., \& Mladenić, D. (2005). D1.7.1 Ontology generation from scratch, SEKT Deliverable. Ljubljana: EU-IST Project IST-2003-506826 SEKT: Semantically Enabled Knowledge Technologies.

Garraway, J. (2010). Knowledge boundaries and boundary-crossing in the design of work-responsive university curricula. Teaching in Higher Education, 211-222.

Gasson, S. (2005). The dynamics of sensemaking, knowledge, and expertise in collaborative, boundary-spanning design. Journal of computer-mediated communication.

Hartwich, F., Pérez, M., Ramos, L., \& Soto, J. (2007). Knowledge management for agricultural innovation: lessons from networking efforts in the Bolivian Agricultural Technology System. Knowledge management for development journal, 21-37.

Hong, J., \& Snell, R. (2015). Boundary-crossing and the localization of capabilities in a Japanese multinational firm. ASIA PACIFIC BUSINESS REVIEW, 19.

Im, G., \& Rai, A. (2008). Knowledge sharing ambidexterity in long-term interorganizational relationships. Management science, 1281 - 1296.

Lee, J., Min, J., \& Lee, H. (2017). Setting a knowledge boundary across teams: knowledge protection regulation for inter-team coordination and team performance. JOURNAL OF KNOWLEDGE MANAGEMENT, 21.

Majchrzak, A., More, P., \& Faraj, S. (2012). Transcending Knowledge Differences in Cross-Functional Teams. RGANIZATION SCIENCE, 20.

Marheineke, M., Habicht, H., \& Moslein, K. (2016). Bridging knowledge boundaries: the use of boundary objects in virtual innovation communities. $R$ \& D MANAGEMENT, 11.

Rau, C., Neyer, A., \& Moslein, K. (2012). Innovation practices and their boundary-crossing mechanisms: a review and proposals for the future. TECHNOLOGY ANALYSIS \& STRATEGIC MANAGEMENT, 37. 
Rehm, S., \& Goel, L. (2015). The emergence of boundary clusters in inter-organizational innovation. INFORMATION AND ORGANIZATION, 25.

Smith, P. (2016). Boundary emergence in inter-organizational innovation The influence of strategizing, identification and sensemaking. EUROPEAN JOURNAL OF INNOVATION MANAGEMENT, 25.

Swart, J., \& Harvey, P. (2011). Identifying knowledge boundaries: the case of networked projects. Journal of Knowledge Management, 703-721.

Tell, F., Berggren, C., Brusoni, S., \& Van de Ven, A. (2017). Manageing Knowledge Integration Across Boundaries. Oxford: Oxford Scholarship Online.

Wannenmacher, D., \& Antoine, A. (2016). Management of innovative collaborative projects: Moments of tension and the Peer-Mediation Process-a case-study approach. KNOWLEDGE MANAGEMENT RESEARCH \& PRACTICE, 11.

Wilhelm, M., \& Dolfsma, W. (2018). Managing knowledge boundaries for open innovation - lessons from the automotive industry. INTERNATIONAL JOURNAL OF OPERATIONS \& PRODUCTION MANAGEMENT, 19.

Young, M., \& Muller, J. (2010). Three Educational Scenarios for the Future: lessons from the SOciology. EUROPEAN JOURNAL OF EDUCATION, 17.

Zarate, P., \& Liu, S. (2016). A new trend for knowledge-based decision support systems design. International Journal of Information and Decision Sciences, 305-324. 